\title{
CORRECTION
}

\section{Correction to "Working in a bubble: How can businesses reopen while limiting the risk of COVID-19 outbreaks?"}

CMAJ has been made aware of an error that occurred in the Nov. 2, 2020, issue. ${ }^{1,2}$

In the author byline, John Brownstein's name had an incorrect middle initial; it should have read John S. Brownstein.

This has been corrected at cmaj.ca.

Cite as: CMAJ 2021 May 3;193:E650. doi: 10.1503/cmaj.210701

\section{References}

1. Shaw J, Day T, Malik N, et al. Working in a bubble: How can businesses reopen while limiting the risk of COVID-19 outbreaks? CMAJ 2020;192: E1362-6.

2. Shaw J, Day T, Malik N, et al. Les bulles de travail : Comment les entreprises peuvent-elles rouvrir en réduisant le risque d'éclosions de la COVID-19? CMAJ 2020;192:E1362-6. 